\title{
Significance of a preoperative tumor marker gradient for predicting microvascular invasion in cases of hepatocellular carcinoma
}

\author{
SATOSHI KOIZUMI ${ }^{1}$, SHINGO YAMASHITA ${ }^{2}$, SATOSHI MATSUMURA ${ }^{3}$, KAZUHISA TAKEDA ${ }^{4}$, \\ TAKUYA MINAGAWA ${ }^{5}$, SHINJIRO KOBAYASHI ${ }^{1}$, TAIZO HIBI $^{5,6}$, MASAHIRO SHINODA ${ }^{5}$, \\ ITARU ENDO ${ }^{4}$, MINORU TANABE $^{3}$, MASAKAZU YAMAMOTO $^{2}$ and TAKEHITO OTSUBO ${ }^{1}$
}

\begin{abstract}
${ }^{1}$ Division of Gastroenterological and General Surgery, St. Marianna University School of Medicine, Kawasaki, Kanagawa 216-8511; ${ }^{2}$ Department of Surgery, Institute of Gastroenterology, Tokyo Women's Medical University, Tokyo 162-8666; ${ }^{3}$ Department of Hepatobiliary and Pancreatic Surgery, Tokyo Medical and Dental University, Tokyo 113-8519; ${ }^{4}$ Department of Gastroenterological Surgery, Yokohama City University, Graduate School of Medicine,

Yokohama 236-0004; ${ }^{5}$ Department of Surgery, School of Medicine, Keio University, Tokyo 160-8582;

${ }^{6}$ Department of Pediatric Surgery and Transplantation, Kumamoto University, Kumamoto 860-8556, Japan
\end{abstract}

Received April 1, 2019; Accepted September 23, 2019

DOI: $10.3892 / \mathrm{mco} .2020 .1975$

\begin{abstract}
Although vascular invasion is an important factor in the progression and treatment of hepatocellular carcinoma (HCC), it remains difficult to determine, on the basis of preoperative imaging alone, whether vascular invasion, especially microvascular invasion, has occurred. The current retrospective study enrolled 292 patients who, between 2004 and 2014, underwent curative hepatectomy as an initial treatment for HCC. The patients were divided between those with $(n=70)$ and those without $(n=222)$ microvascular invasion. Whether tumor-marker-based prediction of microvascular invasion was possible was assessed by comparing the preoperative serum $\alpha$-fetoprotein (AFP) and prothrombin induced by vitamin K absence or antagonist-II concentrations between two groups of patients. The AFP concentration was significantly higher in patients with microvascular invasion compared with patients without microvascular invasion ( $\mathrm{P}=0.0019)$. Stepwise logistic regression analysis demonstrated the AFP concentration and the logarithmic conversion ratio of the AFP gradient $(\log$ AFP grad) to be useful $(\mathrm{P}=0.0019 ; 0.0424)$ for predicting microvascular invasion. The serum AFP concentration and log AFP grad appear to be clinically useful in predicting microvascular invasion in patients with HCC.
\end{abstract}

Correspondence to: Dr Satoshi Koizumi, Division of Gastroenterological and General Surgery, St. Marianna University School of Medicine, 2-16-1 Sugao, Miyamae-ku, Kawasaki, Kanagawa 216-8511, Japan

E-mail: satoshi.koizumi@marianna-u.ac.jp

Key words: tumor marker, $\alpha$-fetoproteingradient, microvascular invasion

\section{Introduction}

In cases of hepatocellular carcinoma (HCC), measurement of tumor markers $\alpha$-fetoprotein (AFP) and prothrombin induced by vitamin $\mathrm{K}$ absence or antagonist-II (PIVKA-II) is widely performed worldwide because such measurement is complementary to imaging for both diagnosis and evaluation of the effects of therapy. In addition, various studies have shown these same tumor markers to be clinically promising in terms of predicting vascular invasion or HCC recurrence (1-9). Determining the presence or absence of microvascular invasion in cases of $\mathrm{HCC}$ is very important when treatment strategies are being considered, but detecting microvascular invasion remains difficult despite advancements in various imaging techniques. Further, whether tumor marker levels can be used to predict the presence of microvascular invasion is unknown. We, as members of the Association for Clinical Research on Surgery, conducted a retrospective study in which we attempted to clarify whether microvascular invasion can be predicted preoperatively on the basis of a tumor marker gradient in patients who have not undergone previous treatment for HCC.

\section{Patients and methods}

Patient selection. Included in the study were 292 patients, each of whom had undergone curative hepatectomy as initial treatment for $\mathrm{HCC}$ at one of the five Association for Clinical Research on Surgery (ACRoS) member hospitals (Tokyo Women's Medical University, Tokyo Medical and Dental University, Yokohama City University, Keio University, or St. Marianna University School of Medicine hospital) between January 2004 and December 2014. Each of these hospitals is a high-volume liver surgery center, and the patients were identified through a search of hospital records. Curative hepatectomy 
was defined as hepatectomy in which all existing tumors were resected macroscopically. Patients included in the study met the following criteria: Treatment was for a solitary tumor $<5 \mathrm{~cm}$ in diameter and diagnosed histopathologically as HCC after surgery, and AFP and/or PIVKA-II was measured more than twice during the 3-month period, with the first measurement obtained at the time of the patient's first visit and the second or last measurement obtained at the time of admission to the hospital for surgery. Patients not included in the study were those for whom vascular invasion was diagnosed by imaging, those who had undergone prior treatment for $\mathrm{HCC}$, those with remarkably low hepatic functional reserve (Child-Pugh score $>8$ points), those who were using warfarin regularly, and those whose decision-making ability was deemed compromised.

The study was approved by the Committee for Medical Ethics and clinical studies at each of the five universities, including that of St. Marianna University School of Medicine (Kawasaki, Japan) (approval no. 2803). Each applicable patient received opt-out consent.

Diagnosis of HCC. The HCC had been diagnosed preoperatively according to the protocol established at each of the five universities. The diagnosis in all cases was based on computed tomography (CT) and magnetic resonance imaging (MRI) findings and on tumor marker concentrations. None of the patients had undergone needle biopsy. In all cases, contrast $\mathrm{CT}$ and/or contrast MRI had been performed, and the number of tumors and tumor diameters were judged from the images obtained.

Measurement of AFP and PIVKA-II. Serum AFP concentrations had been measured by latex agglutination immunoassay (LPIA-A700 kit; Daia-iatron), and serum PIVKA-II concentrations had been measured by enzyme immunoassay with a monoclonal antibody specific for PIVKA-II (PIVKA-II kit; Eisai). A gradient was calculated for each marker (AFP grad and PIVKA-II grad) by dividing the difference between the preoperatively measured concentrations by the number of days between measurements. However, because the gradient variances were large, logarithmic conversion was performed, and the logarithmic conversion ratios (log AFP grad and log PIVKA-II grad) were used for analysis.

Surgery and pathological evaluation. The hepatectomy had been deemed curative in all 292 patients, and there were no in-hospital deaths. Macroscopic tumor type and pathological features (tumor size, vascular invasion, lymph node metastasis, distant organ metastasis) had been assessed according to the General Rules for the Clinical and Pathological Study of Primary Liver Cancer of the Liver Cancer Study Group of Japan (10).

Microvascular invasion was diagnosed definitively when invasion of the tumor into the portal vein, hepatic vein, and/or bile duct was found during postoperative pathological examination of the surgical specimen.

Statistical analysis. Differences in categorical variables, e.g., sex, viral status, and Child-Pugh class, between patients with and without microvascular invasion were analyzed by means of chi-square test or Fisher's exact test, and differences in continuous variables, e.g., markers of liver function, such as the AFP concentration, were analyzed by means of Student's t-test. Association between continuous variables (e.g., age, ICG-R15, tumor size, concentrations of the tumor markers such as AFP and PIVKA-II, and tumor marker grad) and microvascular invasion was tested by means of stepwise logistic regression analysis, and receiver operating characteristic (ROC) curves were drawn to determine optimum cut-off AFP grad and PIVKA-II grad values for predicting microvascular invasion of HCC. JMP software (version 12; SAS Institute Inc.) was used for all statistical analyses, and $\mathrm{P}<0.05$ was considered significant.

\section{Results}

Clinical characteristics of the patients are shown per group in Table I. There was no significant between-group difference in the sex ratio, age, hepatic functional reserve, or viral status. There was a difference, however, in the AFP concentrations.

The postoperative pathological diagnoses are shown per group in Table II. Although small nodular tumors with obscure margins and simple nodular tumors were found in many of the patients with microvascular invasion, there was no significant between-group difference in the macroscopic tumor types or the presence of LN metastasis.

Stepwise logistic regression analysis showed the AFP concentration and log AFP grad to be influential variables. ROC curves obtained for the AFP concentration and log AFP grad are shown in Figs. 1 and 2, respectively. The cut-off values obtained by means of the Youden index were $33 \mathrm{ng} / \mathrm{ml}$ and -0.76214, respectively. Log AFP grad -0.76214 approximates to AFP grad 0.4665 .

\section{Discussion}

Liver cancer is one of the five major cancers that occur in Japan, with HCC accounting for the majority of primary liver cancers (11). Therefore, treatment that improves either prognosis or the quality of life of patients with liver cancer has become an important public policy focus. Treatment of HCC has progressed worldwide, with hepatectomy, ablation, and catheterization currently standing as the pillars of HCC treatment. In selecting the ideal treatment modality for a patient and to obtain the best possible outcome, both the degree of cancer progression and hepatic functional reserve must be taken into consideration. Important factors pertaining to the degree of cancer progression are the number and size of tumors and the presence or absence of microvascular invasion. Newly developed imaging devices and systems and advances in detection methods have made accurate diagnosis possible, but identifying microvascular invasion remains difficult. Formerly, we attempted to determine progression status by predicting pathological factors, particularly vascular invasion, on the basis of the macroscopic type of tumor identified on preoperative CT or MR images $(12,13)$. Microvascular invasion was correctly identified in only $\sim 70 \%$ of cases, however. The treatment strategy for HCC is selected not only on the basis of the cancer stage but also on that of the patient's hepatic functional reserve. Background viral hepatitis often results in poor hepatic functional reserve, and the presence or absence 
Table I. Patient characteristics, per study group.

\begin{tabular}{|c|c|c|c|}
\hline Characteristic & Without vascular invasion $(\mathrm{n}=222)$ & With vascular invasion $(n=70)$ & P-value \\
\hline Sex ratio (male:female) & 168:54 & $54: 16$ & 0.8013 \\
\hline Age [median (IQR) years] & $70(62-74)$ & $70.5(62-75.3)$ & 0.4188 \\
\hline Child-Pugh class & & & 0.2850 \\
\hline A & 210 & 65 & \\
\hline B & 11 & 3 & \\
\hline Unknown & 1 & 2 & \\
\hline ICG-R15 [median (IQR) \%] & $13.31(9-20.1)$ & $16(10-20.6)$ & 0.4777 \\
\hline Viral status & & & 0.2397 \\
\hline HBV & 48 & 14 & \\
\hline $\mathrm{HCV}$ & 110 & 31 & \\
\hline NonB NonC & 64 & 25 & \\
\hline \multicolumn{4}{|l|}{ Tumor markers } \\
\hline $\operatorname{AFP}(\mathrm{ng} / \mathrm{ml})$ & $8(3.8-33)$ & $38.5(6.8-347.3)$ & 0.0019 \\
\hline PIVKA-II (mAu/ml) & $36.5(19-126.8)$ & $77(24.8-417)$ & 0.2825 \\
\hline
\end{tabular}

ICG-R15, indocyanine green retention rate at $15 \mathrm{~min}$; HBV, hepatitis B virus; HCV, hepatitis C virus; AFP, $\alpha$-fetoprotein; PIVKA-II, prothrombin induced by vitamin $\mathrm{K}$ absence or antagonist-II.

Table II. Final pathological diagnoses, per study group.

\begin{tabular}{lccc}
\hline Variables & Without vascular invasion $(\mathrm{n}=222)$ & With vascular invasion $(\mathrm{n}=70)$ & P-value \\
\hline Macroscopic tumor type & 14 & 0 & a \\
Obscure & 139 & 35 \\
SN & 37 & 19 \\
SNEG & 27 & 15 \\
Multinodular & 0 & 1 \\
Massive & 1 & 0 & 0.037 \\
Invasiove & 4 & $3(2.475-3.625)$ & $\mathrm{a}$ \\
Other & $2.7(2-3.5)$ & 1 & \\
Size [median $(\mathrm{IQR}) \mathrm{cm}]$ & & 68 & \\
LN metastasis & 0 & 1 & \\
Positive & 216 & 6 & \\
Negative & 6 & & \\
Unknown & & & \\
\hline
\end{tabular}

Obscure, small nodular type with an obscure boundary; $S N$, simple nodular type; $S N E G$, simple nodular type with extranodular growth pattern; Massive, massive growth pattern; Invasive, invasive growth pattern; $L N$, lymph node; ${ }^{\mathrm{P}} \mathrm{P}-\mathrm{value}$ not obtained because at least $20 \%$ of the expected frequencies were $<5$.

microvascular invasion is an important consideration in determining whether to prioritize curability or hepatic functional reserve. Thus, it is important to ascertain the presence or absence of vascular invasion in the $30 \%$ of cases for which this cannot be done on the basis of images obtained.

Several clinical studies of tumor markers have been reported $(2,3,5-7,9)$. PIVKA-II was shown in these studies to be a useful prognostic factor in cases of HCC unrelated to Hepatitis B virus (HBV) or Hepatitis C Virus (HCV). HCC tumors are known to develop rapidly after microvascular invasion, and PIVKA-II is predicted to increase accordingly. It is from this perspective that we designed our study. Initially, we planned to examine AFP and PIVKA-II doubling time, but we judged doubling time to be an inappropriate variable because variation in this time is great and such times are not normally distributed. For this reason, we examined logarithmic tumor marker (AFP, PIVKA-II) gradient values as predictor variables.

Medical insurance in Japan does not cover measurement of tumor markers more than once within the same month. 


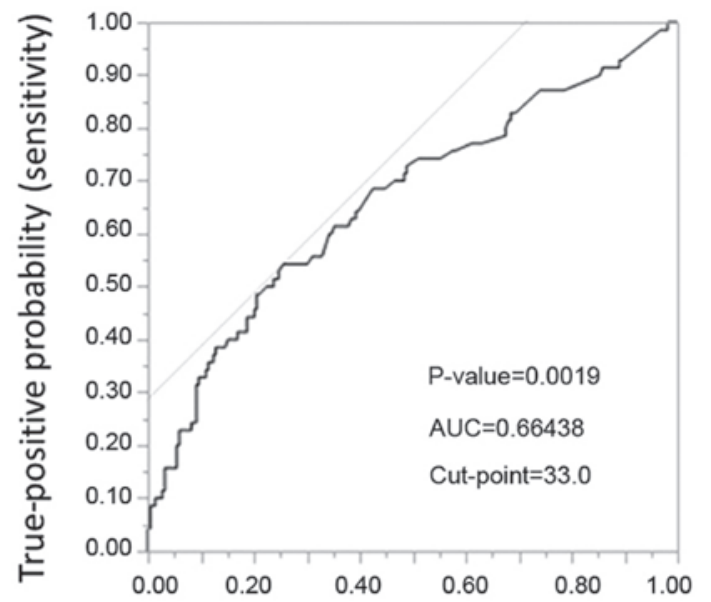

False-positive probability (1-specificity)

ROC curve: receiver operating characteristics curve AUC: area under the curve

Figure 1. ROC curve for AFP-based prediction of microvascular invasion in patients with HCC. ROC, receiver operating characteristic; AFP, $\alpha$-fetoprotein; HCC, hepatocellular carcinoma

Therefore, gathering a suitable number of cases for this study was expected to be difficult. To solve this problem, we identified pertinent cases treated over a period of 10 years at each of five high-volume centers, and the number of cases that we included $(\mathrm{n}=292)$ allowed for valid statistical comparisons.

Of all clinical factors that we investigated, only tumor size and AFP values differed significantly between patients with and without microvascular invasion of the HCC. We cannot clearly explain why AFP rather than PIVKA-II was found to be associated with microvascular invasion, and we did not investigate the underlying mechanism responsible for this association. However, the proportion of patients with an AFP concentration higher than the reference value was greater than the proportion of patients with a PIVKA-II concentration higher than the reference value, and this might, at least in part, explain our finding. Because we assumed when we designed the study that the size and number of tumors are related to microvascular invasion, we included only cases of a solitary tumor $<5 \mathrm{~cm}$. Significant differences in microvascular invasion were observed under these conditions, so we can say with confidence that the size of the tumor is an important factor in judging whether microvascular invasion has occurred. Even so, as indicated above, median size of the tumors was quite similar between our two groups, and thus it cannot be said that tumor size is a useful indicator of microvascular invasion when the diameter is around $3 \mathrm{~cm}$. However, we identified cut-off values for AFP and $\log$ AFP grad $(33 \mathrm{mg} / \mathrm{ml}$ and $-0.76214 \mathrm{ng} / \mathrm{ml}$, respectively) that can be used to predict the presence or absence of microvascular invasion at $\geq 60 \%$ accuracy. Use of a logarithmic value may seem cumbersome, but log AFP grad -0.76214 approximates to AFP grad 0.466, so the problem of practicality is easily resolved. When AFP increases by $5 \mathrm{ng} / \mathrm{ml}$ or more within 10 days, it can be taken as an indicator of microvascular invasion with a predictive accuracy of $60 \%$. Use of the cut-off values of both AFP and $\log$ AFP grad yields a predictive accuracy of $\sim 70 \%$ in cases in

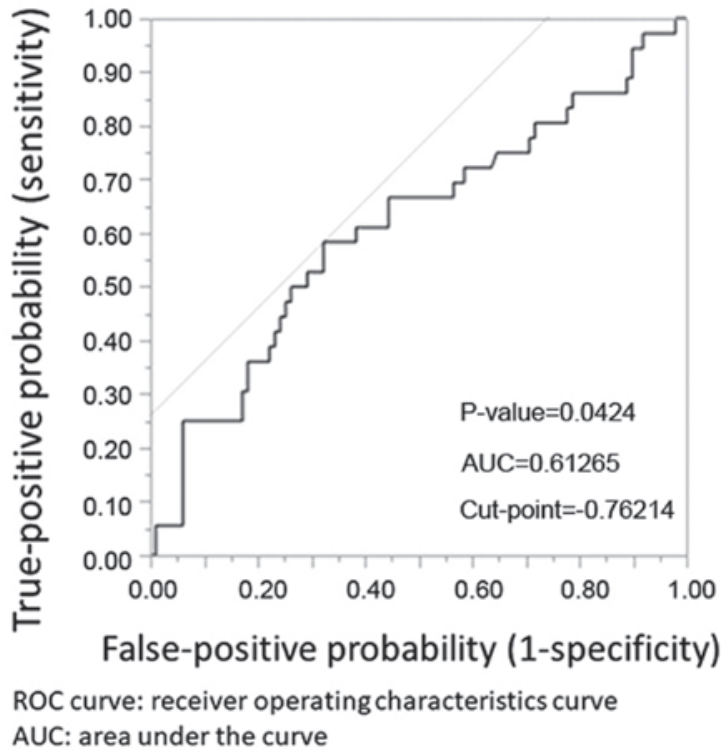

Figure 2. ROC curve for log AFP grad-based prediction of microvascular invasion in patients with HCC. ROC, receiver operating characteristic; AFP, $\alpha$-fetoprotein; HCC, hepatocellular carcinoma.

which imaging was not informative with respect to microvascular invasion.

Overall, our study data suggest that the presence or absence of microvascular invasion in cases of HCC can, before surgery, be predicted on the basis of the AFP concentration and AFP gradient when these are used in conjunction with preoperative imaging. We note however, that that some patients will have an HCC that does not secrete AFP, and thus prediction of microvascular invasion on the basis of this tumor marker will not be possible in all cases.

\section{Acknowledgements}

The authors would like to thank Professor Eisuke Inoue of the Department of Medical Education at St. Marianna University School of Medicine for providing advice regarding the statistical analysis performed in this study. Authors would also like to thank Professor Tina Tajima of the Department of Medical Education at St. Marianna University School of Medicine for advice regarding the proofreading of this manuscript.

\section{Funding}

No funding was received.

\section{Availability of data and materials}

The datasets used and/or analyzed during the present study are available from the corresponding author on reasonable request.

\section{Authors' contributions}

SKoi conceived and designed the research. SKoi, SY, SM, KT, TM, TH and SKob performed data acquisition, data analysis and manuscript preparation. MS, IE, MT, MY and TO assisted 
with data analysis and statistical analysis. The final version of the manuscript has been approved by all authors.

\section{Ethics approval and consent to participate}

The study was approved by the each Ethics Committee of ACRoS member's hospital.

\section{Patient consent for publication}

Not applicable.

\section{Competing interests}

The authors declare that they have no competing interests.

\section{References}

1. Kaibori M, Ishizaki M, Matsui K and Kwon A: Clinicopathologic characteristics of patients with non-B non- $\mathrm{C}$ hepatitis virus hepatocellular carcinoma after hepatectomy. Am J Surg 204: 300-307, 2012

2. Kaibori M, Matsui Y, Yanagida H, Yokoigawa N, Kwon AH and Kamiyama Y: Positive status of alpha-fetoprotein and des-gamma-carboxy prothrombin: Important prognostic factor for recurrent hepatocellular carcinoma. World J Surg 28: 702-707, 2004.

3. Alejandro AG and Cervantes JG: Diagnostic value of des-gamma carboxy prothrombin as compared with alpha feto protein in hepatocellular carcinoma: A meta-analysis. J Gastroenterol 2: 67-73, 2006.

4. Kaibori M, Saito T, Matsui Y, Uchida Y, Ishizaki M and Kamiyama Y: A review of the prognostic factors in patients with recurrence after liver resection for hepatocellular carcinoma. Am J Surg 193: 431-437, 2007.
5. Inagaki Y, Tang W, Makuuchi M, Hasegawa K, Sugawara Y and Kokudo N: Clinical and molecular insights into the hepatocellular carcinoma tumour marker des- $\gamma$-carboxyprothrombin. Liver Int 31: 22-35, 2011.

6. Kaibori M, Ishizaki M, Matsui K and Kwon AH: Predictors of microvascular invasion before hepatectomy for hepatocellular carcinoma. J Surg Oncol 102: 462-468, 2010.

7. Hemida K, Kamal W, Riad GS, Adel NA, Ayoub MS and Fekry D: Evaluation of Pivka-II as a predictor marker for portal vein obstruction in hepatocellular carcinoma patients. J Am Sci 8: 162-170, 2012.

8. Hirokawa F, Hayashi M, Miyamoto Y, Asakuma M, Shimizu T, Komeda K, Inoue $\mathrm{Y}$ and Uchiyama K: Outcomes and predictors of microvascular invasion of solitary hepatocellular carcinoma. Hepatol Res 44: 846-853, 2014.

9. Zakhary NI, Khodeer SM, Shafik HE, Camelia A and Abdel Malak CA: Impact of PIVKA-II in diagnosis of hepatocellular carcinoma. J Adv Res 4: 539-546, 2013.

10. Liver Cancer Study Group of Japan: The general rules for the clinical and pathological study of primary liver cancer 2nd edition. Kanehara \& Co., Ltd., Tokyo, 2003.

11. Cancer Registry and Statistics: Cancer information service, National cancer center. Japan, 2017.

12. Koizumi S, Kobayashi S, Asakura T, Nakano H, Morimoto T, Koike J and Otsubo T: Preoperative diagnosis of macroscopic type of hepatocellular carcinoma by multi-detector-row computed tomography is useful for prediction of the occurrence of pathological progression factors in the tumor. J St. Marianna Univ 2: 9-16, 2011

13. Ariizumi S, Kitagawa K, Kotera Y, Takahashi Y, Katagiri S, Kuwatsuru R and Yamamoto M: A non-smooth tumor margin in the hepatobiliary phase of gadoxetic acid disodium (Gd-EOB-DTPA)-enhanced magnetic resonance imaging predicts microscopic portal vein invasion, intrahepatic metastasis, and early recurrence after hepatectomy in patients with hepatocellular carcinoma. J Hepatobiliary Pancreat Sci 18: 575-585, 2011. 